\title{
ASPECTOS RELACIONADOS AO PRÉ-NATAL E PARTO E ÓBITO NEONATAL PRECOCE
}

\author{
ASPECTS RELATED TO PRENATAL AND CHILDBIRTH AND EARLY \\ NEONATAL DEATH
}

\author{
Deyziane Fernandes da Silva ${ }^{1}$ \\ Vitória Keller Gregório de Araújo ${ }^{2}$ \\ Erianna Yadja Lucina de Macedo ${ }^{3}$ \\ Giovana Souza D'oleron Barreto ${ }^{4}$ \\ Nilba Lima de Souza ${ }^{5}$
}

\begin{abstract}
RESUMO: OBJETIVO: Analisar os aspectos relacionados ao pré-natal e parto e a ocorrência de óbitos neonatal precoce. MÉTODO: Trata-se de um estudo retrospectivo descritivo, de caráter quantitativo realizado em uma maternidade pública de ensino no qual foram analisados todos os prontuários de puérperas e de seus respectivos recém-nascidos que tiveram óbito neonatal precoce no período que vai de 2014 a 2017. A coleta de dados foi realizada com um instrumento semiestruturado que incluíam variáveis maternas. Foram analisados 81 prontuários da puérpera e recém-nascidos com registro de óbito neonatal precoce. RESULTADOS: Foram registrados_destes 23(vinte e três) óbitos em 2014, 7(sete) óbitos, em 2015, 20 (vinte) em 2016 e 31(trinta e um) em 2017. A média de consultas pré-natal, quando relacionada às semanas gestacional na qual ocorreu o parto e o nascimento foi considerado insatisfatório em quantidade quando comparado ao padrão preconizado pelo Ministério da saúde para cada escore, porém há de se considerar o número de parto cesáreo em cada ano de investigação e o número de óbito neonatal precoce. CONCLUSÃO: Os resultados apontam um número de consultas pré-natal insatisfatório e um número de parto cesáreo elevado como fatores que podem favorecer a ocorrência de óbitos neonatal precoce.
\end{abstract}

Palavras chaves: Pré-natal. Saúde Materno-Infantil. Morte Perinatal.

\footnotetext{
1 Graduanda do curso de enfermagem na Universidade Federal do Rio grande do Norte;

2 Graduanda do curso de enfermagem na Universidade Federal do Rio grande do Norte;

${ }^{3}$ Enfermeira formada na Universidade Federal do Rio Grande do Norte;

${ }^{4}$ Graduanda do curso de enfermagem na Universidade Federal do Rio grande do Norte;

${ }^{5}$ Professora doutora no departamento de enfermagem da UFRN.
} 
ABSTRACT: OBJECTIVE: To analyze aspects related to prenatal and childbirth and the occurrence of early neonatal deaths. METHOD: This is a retrospective, descriptive, quantitative study carried out in a public teaching maternity in which all the medical records of puerperal women and their respective newborns who had early neonatal death from 2014 to 2017 were analyzed. Data collection was carried out with a semi-structured instrument that included maternal variables. 81 medical records of the puerperal woman and newborns with an earlyneonatal death record were analyzed. RESULTS: Of these 23 (twenty-three) deaths were recorded in 2014, 7 (seven) deaths in 2015, 20 (twenty) in 2016 and 31 (thirty-one) in 2017. The average number of prenatal consultations, when related to the gestational weeks in which the delivery took place and the birth was considered unsatisfactory in quantity when compared to the standard recommended by the Ministry of Health for each score, however the number of cesarean deliveries in each year of investigation and the number of neonatal deaths must be considered precocious. CONCLUSION: The results indicate an unsatisfactory number of prenatal consultations and a high number of cesarean deliveries as factors that may favor the occurrence of early neonatal deaths.

Keywords: Prenatal. Maternal and Child Health. Perinatal Death. 


\section{INTRODUÇÃO}

A mortalidade materno-infantil é um importante indicador do desenvolvimento de um país, de modo que quanto maior forem as taxas de morbimortalidade menor será o índice desenvolvimento do país. No Brasil, o combate às iniquidades em saúde dessa ordem já vem sendo promovidas há algum tempo.

Há de se considerar também a mortalidade materna. Aproximadamente 830 mulheres morrem todos os dias por causas evitáveis relacionadas à gestação e ao parto no mundo, $99 \%$ de todas as mortes maternas ocorrem em países em desenvolvimento como o Brasil, a mortalidade materna é maior entre mulheres que vivem em áreas rurais e comunidades mais pobres, assim como a mortalidade infantil (OPAS, 2018). Forças de ordem política, social e econômica influenciam na maneira com as pessoas vivem, adoecem e morrem. A desigualdade na distribuição de poder, rendimentos, bens e serviços favorecem a exposição das pessoas a diferentes riscos e vulnerabilidades (FONSECA et. al., 2019).

Em 2006, como estratégia para consolidação do SUS, foi criado o pacto pela saúde em que seu componente pacto pela vida estabelecia ação prioritária no campo da saúde que deverá ser executada com foco em resultados, e entre as prioridades está a redução da mortalidade materno-infantil, Em 2011 foi instituída a Rede Cegonha, consistindo numa rede de cuidados que visa assegurar à mulher o direito ao planejamento reprodutivo e à atenção humanizada na gravidez, ao parto e ao puerpério, assim como à criança o direito ao nascimento seguro e ao crescimento e desenvolvimento saudáveis, tendo como um de seus princípios a redução a mortalidade materna e infantil com ênfase no componente neonatal (LEAL, 2018; SANINE, 2019; BOZATSKI, 2019).

A Política Nacional de Atenção Integral à Saúde da Criança (PNAISC) em 2015, objetivou promover e proteger a saúde da criança e o aleitamento materno, mediante a atençãoe cuidados integrais e integrados da gestação até aos 9 (nove) anos de vida, com atenção especial à primeira infância e às populações de maior 
vulnerabilidade, tendo em vista à redução da morbimortalidade e um ambiente com condições dignas para o pleno desenvolvimento. Essas e outras políticas retratam o esforço para melhorar os indicadores de saúde materno infantil no Brasil (ALVES et. al., 2018).

Destaca-se o pré-natal como uma ferramenta importantíssima no acompanhamento de uma gestação, visto que neste período é possível identificar precocemente agravos à saúde da gestante e feto, aumentando assim as chances da gravidez resultar em um parto com mãe e recém-nascidos vivos e saudáveis (GARCIA et. al., 2019).

A realização do pré-natal representa papel fundamental não só pela detecção precoce de doenças, mas também por seu trabalho de preparar a mulher para a maternidade. No decorrer das consultas, os profissionais de saúde realizam ações educativas sobre o parto e o cuidado da criança (puericultura), além de orientações essenciais sobre hábitos de vida e higiene pré-natal, além do estado nutricional adequado, modificações fisiológicas do corpo durante o processo de gestação e destaca também sinais e sintomas de alerta para as intercorrencias na ciclo gravídico e puerperal. Estes são apenas alguns dos muitos benefícios encontrados no acompanhamento pré-natal (GARCIA et. al., 2019).

No Brasil, os nascimentos ocorrerem predominantemente em hospitais $(98,4 \%)$ e $(88,7 \%)$, com assistência predominante de médicos. Porém os resultados brasileiros são insatisfatórios se comparados a outros países no mundo que atingem taxas menores de mortalidade neonatal e infantil (LANSKY et. al., 2014).

Acredita-se que as intervenções excessivas, por vezes desnecessárias, durante o trabalho de parto pode favorecer a mortalidade materna e neonatal. A intensa medicalizaçãodo parto e nascimento com manutenção de taxas elevadas de morbimortalidade materna e perinatal, possivelmente está relacionada à baixa qualidade da assistência prestada e de práticas obsoletas e iatrogênicas, que podem repercutir sobre os resultados perinatais. Neste contexto, a taxa elevada de cesariana do Brasil é um dos exemplos de maior destaque, chegando a $53,7 \%$ dos nascimentos em 2011(LANSKY et. al., 2014). A mortalidade neonatal no Brasil é 9,4 em 2015 na última analise do ODS (Objetivos para o desenvolvimento sustentável). 
O óbito neonatal é aquele que ocorre antes de 28 semanas de vida e tem a seguinte classificação: neonatal precoce (ocorre até 6 dias de vida) e tardio até (de sete a 28 dias) Frente ao elevado número de óbitos neonatais, em especial o óbito precoce e por considerar como pressuposto do estudo que os óbitos neonatais precoce estão relacionados as condições do pré-natal e parto questiona-se: Há relação entre os óbitos neonatais precoce e os aspecto relacionados ao pré-natal e parto? Desse modo, objetiva-se no presente estudo analisar os aspectos relacionados ao pré-natal e parto e a ocorrência de óbitos neonatal precoce.

\section{MÉTODOS}

Trata-se de um estudo retrospectivo descritivo, de caráter quantitativo realizado em uma Maternidade pública de ensino no estado do Rio Grande do Norte. Foram analisados os prontuários de puérperas e recém-nascido com registro de óbito neonatal precoce no período correspondente a 2014 a 2017.

A coleta de dados ocorreu no período de dezembro de 2019 e janeiro de 2020 por meio de instrumento semiestruturado que contém variáveis maternas e neonatais.

Foram analisados 81 prontuários cujos critérios de inclusão foram prontuários de puérperas em que seus recém-nascidos tiveram registro de óbito neonatal precoce

Os dados foram registrados e analisados com auxílio do Microsoft Excel.

O estudo teve aprovação do Comitê de Ética em Pesquisa da Universidade Federal do Rio Grande do Norte, sob o número 3.825.533. 


\section{RESULTADOS}

Houve registro de 81 óbitos neonatais no intervalo de tempo investigado 23 (vinte e três) óbitos em 2014, 7 (sete) em 2015, 20 (vinte) em 2016 e 31 (trinta e um) em 2017. Para investigar a relação entre os óbitos e as consultas pré-natais das mães dos RN foi calculado a média de consultas realizadas pelas gestantes, conforme a idade gestacional na qual ocorreu oparto, considerando um escore 20 à 25 semanas, de 26 à 30 semanas, de 31 à 35 semanas e de 36 a 41 semanas de gestação em cada ano investigado, cujos dados são apresentados na figura1.

Considerando a idade gestacional e o período em que ocorreu o parto a figura 1 revela que o número de consultas pré-natais foi considerado insatisfatório em quantidade quando comparado ao padrão preconizado pelo Ministério da saúde para cada escore utilizado.

Figura 1- Média de consultas pré-natais de mulheres cujos Recém-nascidos tiveram óbitoneonatal precoce no período de 2014 à 2017. Natal/RN, 2020.

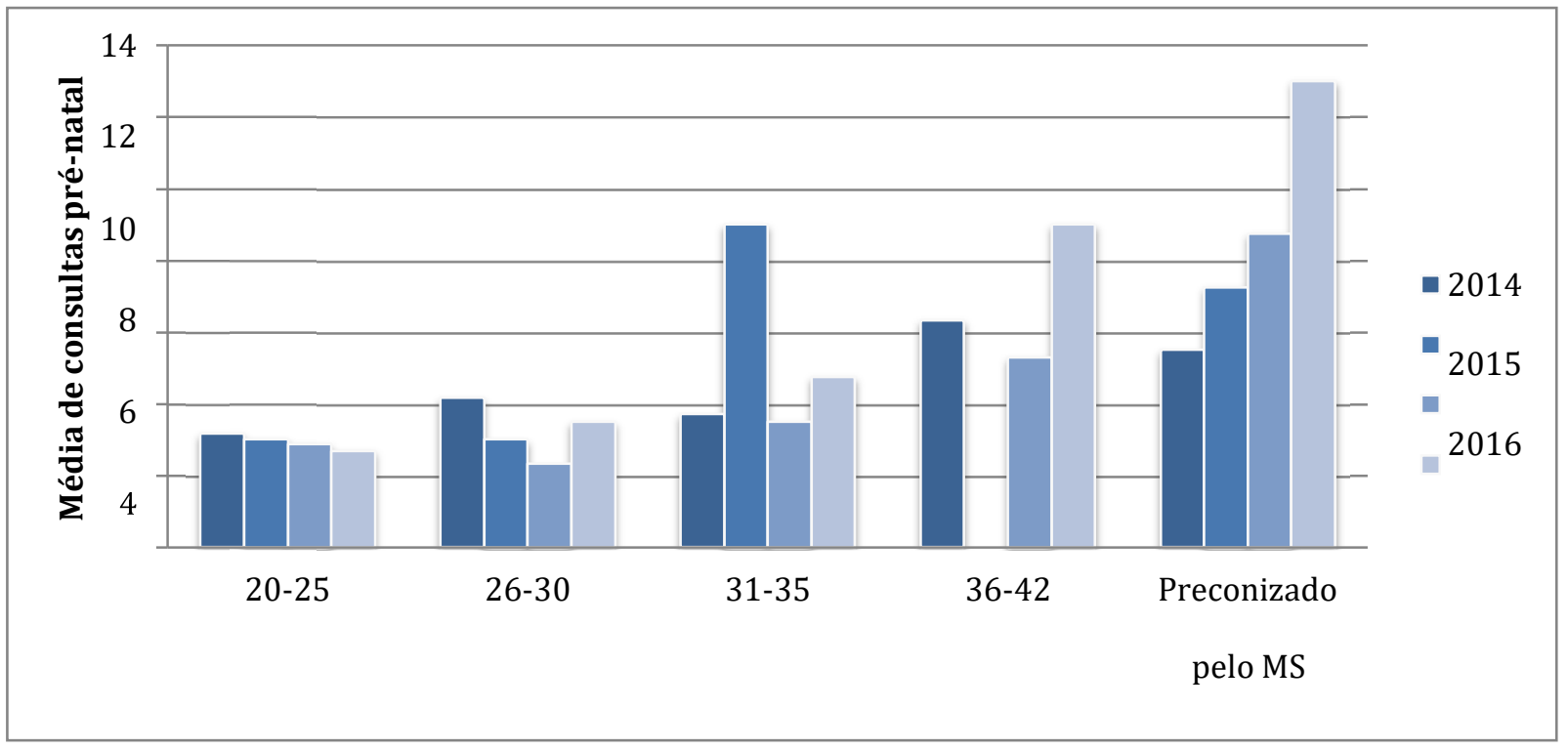

Fonte: Própria pesquisa. 
Buscou-se investigar o tipo de parto e por meio da Figura 2 é possível observar que a ocorrência de parto Cesária em todos os anos de ocorrência dos óbitos foi bem superior a departos vaginais.

Figura 2- Tipo de resolução do parto e nascimento de recém-nascidos com registro de óbitosneonataisprecoce de 2014-2017. Natal/RN, 2020.

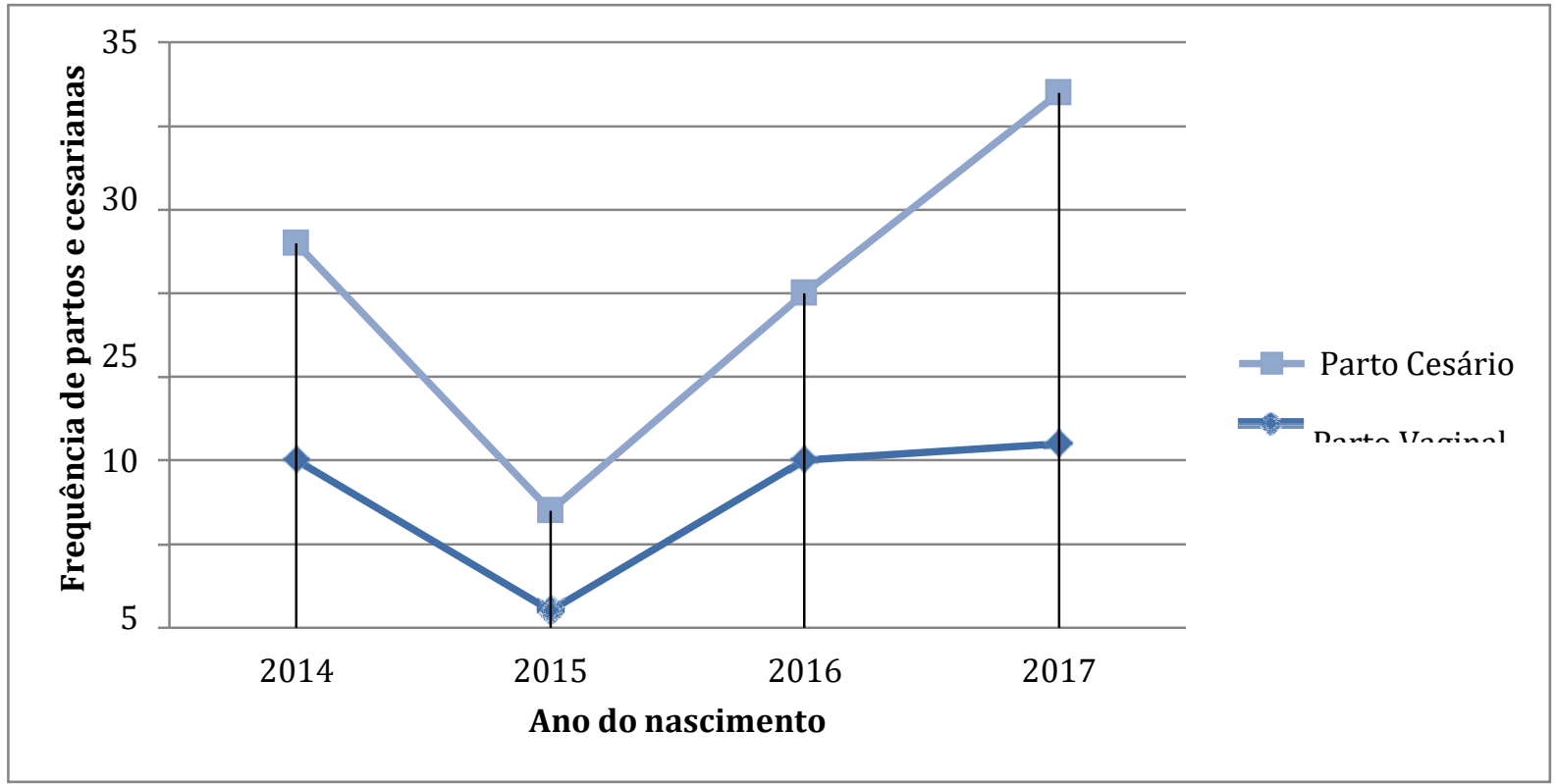

Fonte: Própria pesquisa

Assim na Figura 2, evidencia-se um elevado numero de parto Cesário em relação ao parto normal. Há de se considerar que a instituição campo de investigação é referência para o atendimento de gestante com alto risco, situação que pode favorecer a indicação de parto cesária, quando em situação de alto risco materno-fetal. Porém os dados sugerem investigações mais aprofundadas sobre os resultados apresentados.

A cesariana é um procedimento cirúrgico que com indicação médica adequada tem grande potencial benéfico para parturiente recém-nascido, no entanto o crescente número de partos cesáreos tem se tornado uma preocupação para saúde pública, pois a realização indiscriminada deste procedimento está relacionada com ao aumento de complicações e morte maternas e fetais, de tal modo que os 
países somam esforços para diminuir as taxas de interrupção programadas da gravidez sem indicação médica. Apesar da importância inegável desse procedimento cirúrgico, gestantes e profissionais da saúde necessitam entender os riscosmaternos associados aos distintos modos de realização do parto, utilizando as melhores evidências para evitar possíveis agravos a saúde (MASCARELLO, 2017; NAKANO, 2017).

Em relação às intercorrências no período gestacional a Tabela 1, apresenta as principais delas na qual se observa a prevalência das:

Tabela 1- Intercorrências registradas durante a gravidez e parto nas mulheres investigadas Natal/RN,2020.

\begin{tabular}{|c|c|c|c|c|c|}
\hline & Diabetes & Oligoidrâmnio & Polidrâmnio & SHG & ITU \\
\hline 2014 & $n=1(4 \%)$ & $n=5(22 \%)$ & $n=1(4 \%)$ & $\mathrm{n}=2(9 \%)$ & $n=9(39 \%)$ \\
\hline 2015 & $0 \%$ & $n=1(14 \%)$ & $0 \%$ & $\mathrm{n}=4(57 \%)$ & $n=1(14 \%)$ \\
\hline 2016 & $n=1(5 \%)$ & $n=3(16 \%)$ & $0 \%$ & $n=5(26 \%)$ & $n=2(11 \%)$ \\
\hline 2017 & $n=3(10 \%)$ & $n=4(13 \%)$ & $n=3(10 \%)$ & $\mathrm{n}=6(19 \%)$ & $n=2(6 \%)$ \\
\hline
\end{tabular}

Legenda: SHG (Síndromes hipertensivas da Gestação); ITU (Infecção do trato urinário).

Fonte: própria da pesquisa

Cabe destacar que o trabalho de parto prematuro foi registrado em todos os anos de investigação com maior predominância no ano de 2014 no qual foram registrados $\mathrm{N}(87 \%)$, seguidos de 55\% e 53\% em 2017 e 2016 respectivamente e menor ocorrência em 2015 com N( 43\%) Verifica-se também na Tabela 1 que o diabetes, alterações na quantidade de líquido amniótico, DHEG e infecção de trato urinário são situações registrada em quase todos os anos da investigação o que pode ter favorecido o registro do trabalho de parto prematuro e consequentemente maior risco de mortalidade neonatal. 


\section{DISCUSSÃO}

Em 2000, a ONU aprovou um documento intitulado "Declaração do Milênio", um pacto realizado por 192 países, incluindo o Brasil, que determinaram o compromisso de garantir o desenvolvimento sustentável do planeta. Neste documento constam oito metas a serem atingidas pelos países até 2015 , as "Metas de Desenvolvimento do Milênio" destas metas a $4^{\text {a }}$ é até 2015 , reduzir a mortalidade na infância a dois terços do nível de 1990, a $5^{\text {a }}$ é reduzir a mortalidade materna a três quartos do nível observado em 1990, até 2015 (BRASIL, 2014).

O Brasil vem empenhando-se para diminuir suas taxas de morbimortalidade materno infantil, com esforços para melhorar a qualidade da atenção à saúde e melhor qualidade de vida da população, isso resultou na diminuição das taxas de mortes infantis passado de 53,7 em 1990 para 17,7 óbitos por mil nascidos vivos em 2011, no entanto o componente neonatal não teve o mesmo decréscimo, constitui-se o mais representativo na mortalidade infantil (BRASIL, 2014).

Como mostra os dados da pesquisa, em nenhum dos anos analisados houve um número de consultas pré-natal esperado para a idade gestacional, portanto inferior ao recomendado pelo Ministério da Saúde. Isto representou um risco para a saúde da mulher assim como para a criança, pois se o acompanhamento for realizado de forma adequada a detecção precoce de doenças e o tratamento dos agravos garantem maiores chances de êxito na gravidez (SANTOS et. al., 2018). Além disso a mortalidade neonatal precoce está diretamente relacionada à assistência pré natal e condições de parto e o alto número de mortes maternas reflete desigualdades no acesso aos serviços de saúde e destaca e realça a o paradoxo social (BRASIL, 2014).

O predomínio do parto cesáreo em relação ao parto normal. Sabe-se que quando bem indicado o parto cesáreo é um recurso importante para a redução da mortalidade materna e neonatal, mas que, quando usado de maneira excessiva, pode estar associado a um risco aumentado de resultados maternos desfavoráveis. 
Os motivos relacionados ao aumento considerável nas taxas de cesarianas verificado nos últimos anos no mundo e no Brasil são inúmeros e complexos, podendo-se citar a inadequada organização da rede de atenção à saúde, a falta de qualidade na atenção à saúde relacionada ao parto e fatores socioculturais (MARIN et. al., 2019). Acredita-se que como a instituição campo de pesquisa é um hospital de referência estadual para gravidez e nascimento de alto risco é provável que as mulheres integrantes desse grupo de investigação possam fazer parte do grupo de indicação para parto cirúrgico, embora seja necessária outras investigações que comprovem as suposições feitas. Os agravos apresentados durante a gravidez como o diabetes, a DHEG, alterações na quantidade do líquido amniótico e as ITU identificadas no presente estudo, presente em todos os anos estudados, podem ter influenciado a ocorrência freqüente de trabalho de parto prematuro e as condições de maior gravidade para o recém-nascido, inclusive o óbito. Destaca-se entre essas a ITU como um dos agravos de maior causa de nascimento prematuros. As infecções de trato urinário podem causar, dentre outras coisas, complicações como trabalho de parto e parto pré-termo, ruptura prematura de membranas amniótica, que pode resultar em um recém-nascido de baixo peso dada a restrição de crescimento intrauterino, retardo mental e óbito perinatal e aumento de mortalidade fetal (DUARTE et. al., 2008).

As Síndromes hipertensivas da Gestação (SHG) também foi registrada em todos os anos investigado como um importante indicador de complicações maternas e fetais. As síndromes hipertensivas são as complicações mais frequentes na gestação e constitui, no Brasil, a primeira causa de morte materna, principalmente quando se instalam nas formas graves como a eclâmpsia (KERBER, 2017; OLIVEIRA, 2017).

Ressalta-se que as SHG e ITU são situações detectadas e tratadas durante o pré-natal oque revela que a baixa frequência ao pré-natal identificada neste estudo foi um dos fatores que favoreceu uma evolução insatisfatória da gravidez e as complicações no período perinatal. A assistência pré natal é necessária para identificar de forma precoce fatores de risco maternos e neonatais, e com isso, realizar o tratamento de forma mais efetiva e controlar possíveis dificuldades na saúde da materno infantil. A importância dessa assistência pré natal é o 
acompanhamento da mãe e do desenvolvimento fetal, redução de eventos adversos como por exemplo, toxemia gravídica, que é uma doença que atinge múltiplos sistemas da gestantee pode chegar a uma SHG (Síndrome Hipertensiva Gravídica) dependendo da manifestação clínica e adversas como: alterações no líquido amniótico, diabetes gestacional, entre outras. É necessário a comunicação entre a equipe de saúde, possibilidade de diagnóstico, ter acessoaos exames e com isso, aumentará o número de mulheres que terão um pré natal com qualidade e que evitará complicações fetais (KERBER, 2017; OLIVEIRA, 2017).

As alterações na quantidade de líquido amniótico evidenciada nesta investigação são decorrentes de complicações gestacionais. O polidrâmnio (excesso de líquido amniótico no saco amniótico) está relacionado a problemas como o parto prematuro e descolamento prematuro da placenta e dificulta a respiração materna, isso pode aparecer devido a complicações referentes a SHG (Síndrome Hipertensiva Gestacional) e diabetes gestacional. Cita-se também o oligoidrâmnio (acentuada redução do volume de líquido amniótico), que possui a pré-eclâmpsia como uma doença materna gestacional que está relacionada a essa alteração, e acrescenta-se também, outros eventos prejudiciais como malformações fetais podendo chegar até um óbito fetal. Nesses dois casos de alterações de líquido amniótico, ressalta-se a importância do pré natal, para detectar doenças maternas de forma precoce (ARAÚJO et. al., 2014).

\section{CONCLUSÕES}

Neste estudo identificou-se que os óbitos neonatais precoce nos anos de 2014 à 2017 tiveram no perfil materno mulheres com baixa frequência ao pré-natal, predomínio do parto cesário e complicações detectáveis e controláveis durante a gravidez como ITU, diabetes e SHG. Tais complicações podem ter favorecido as alterações no líquido amniótico, o trabalho de parto prematuro e consequentemente complicações no recém-nascido que favoreceram o óbito neonatal precoce (ARAÚJO, 2014; VARELA, 2017). 
As limitações do estudo refere-se ao seu desenho retrospectivo como coleta de dados secundários, o que requer outras investigações para investigações de outros fatores favoráveis a presente investigação denota a importância da atenção básica sobretudo do pré-natal, no qual tem como objetivo a promoção à saúde da gestante, e o diagnóstico, tratamento e a prevenção das complicações do ciclo gravídico e puerperal. Fatores indispensáveis para redução da mortalidade materna e neonatal. Há de se considerar a importância do vínculo a serfortalecido entre o profissional de saúde e a gestante no acolhimento, incentivo e respeito para que se consiga maior adesão ao pré-natal.

ALVES, Jakeline Barbara et al. Sepse neonatal: mortalidade em município do sul do brasil, 2000 a 2013. Revista Paulista de Pediatria, v. 36, n. 2, p. 132-140, 2018.

\section{REFERÊNCIAS BIBLIOGRÁFICAS}

ARAÚJO, F. G., et. al., Assistência de pré atal na percepção de mães de prematuros. Revista de Enfermagem da UFPE online, v. 08, n. 8, 2014.

BRASIL, Ministério da Saúde. Relatório 2014 para o desenvolvimento do milênio. Disponível em: <https://www.ipea.gov.br/portal/images/stories/PDFs/140523_relatorioodm.pdf>. Acesso em: 19 fev. 2020.

BOZATSKI, Barbara Louise; PINTO, Maria Fernanda; LAVADO, Mylene Martins. Perfil epidemiológico de gestantes diabéticas no município de Itajaí, SC. Arquivos Catarinenses de Medicina, v. 48, n. 2, p. 34-55, 2019.

DUARTE, Geraldo et al. Infecção urinária na gravidez. Revista Brasileira de Ginecologia e Obstetrícia, v. 30, n. 2, p. 1-8, 2008.

Folha Informativa - mortalidade materna. OPAS BRASIL, 2018. Disponível em: https://www.paho.org/bra/index.php?option=com_content\&view=article\&id=5741:folhainformativa-mortalidade-materna\&ltemid=820>. Acesso em: 19 fev. 2020.

FONSECA, R. M. M., et. al., Tendências Associadas a natimortalidade em uma maternidade escola na zona norte de São Paulo. Revista Brasileira de Ginecologia e Obstetrícia, n. 10, v. 41, pág. 1-10, 2019.

GARCIA, Érica Marvila et al. Risco gestacional e desigualdades sociais: uma relação possível? Revista Brasileira de Ginecologia e Obstetrícia, n. 10. v. 41, p. 1-10, 2019.

GONZAGA, Isabel Clarisse Albuquerque et al. Atenção pré-natal e fatores de risco associados à prematuridade e baixo peso ao nascer em capital do nordeste brasileiro. Ciência \& Saúde Coletiva, v. 21, p. 1965-1974, 2016.

KERBER, Guenevere de Franceschi; MELERE, Cristiane. Prevalência de síndromes hipertensivas gestacionais em usuárias de um hospital no sul do Brasil. Revista Cuidarte, v. 8, 
n. 3, p. 1899-1906, 2017.

LANSKY, Sônia et al. Pesquisa Nascer no Brasil: perfil da mortalidade neonatal e avaliação da assistência à gestante e ao recém-nascido. Cadernos de Saúde Pública, v. 30, p. S192-S207, 2014.

LEAL, Maria do Carmo et al. Saúde reprodutiva, materna, neonatal e infantil nos 30 anos do Sistema Único de Saúde (SUS). Ciência \& Saúde Coletiva, v. 23, p. 1915-1928, 2018.

MARIN, Daniela Ferreira D.'Agostini et al. Intervenções direcionadas à redução da taxa de cesarianas no Brasil. Revista Brasileira de Epidemiologia, v. 22, p. e190066, 2019.

MASCARELLO, Keila Cristina; HORTA, Bernardo Lessa; SILVEIRA, Mariângela Freitas. Complicações maternas e cesárea sem indicação: revisão sistemática e meta-análise. Revista de Saúde Pública, v. 51, p. 105, 2017.

NAKANO, Andreza Rodrigues; BONAN, Claudia; TEIXEIRA, Luiz Antônio. O trabalho de parto do obstetra: estilo de pensamento e normalização do "parto cesáreo" entre obstetras. Physis: Revista de Saúde Coletiva, v. 27, p. 415-432, 2017.

OLIVEIRA, Gleica Sodré et al. Assistência de enfermeiros na síndrome hipertensiva gestacional em hospital de baixo risco obstétrico. Revista Cuidarte, v. 8, n. 2, p. 1561-1572, 2017.

SANINE, Patricia Rodrigues et al. Atenção ao pré-natal de gestantes de risco e fatores associados no Município de São Paulo, Brasil. Cadernos de Saúde Pública, v. 35, p. e00103118, 2019.

SANTOS, Leidiene Ferreira et al. Características do pré-natal na perspectiva de mulheres atendidas em Unidades de Atenção Primária à Saúde. Revista de Enfermagem UFPE online, n. 02 , v. 12, p. 1-8, 2018.

TROMBETTA, Julia Balbinotti et al. Fatores associados à qualidade de vida em gestantes de alto risco. 2019.

VARELA, Patrícia Louise Rodrigues et al. Intercorrências na gravidez em puérperas brasileiras atendidas nos sistemas público e privado de saúde. Revista Latino-Americana de Enfermagem, v. 25, 2017. 